\title{
Contribution of Yeast and Plant Research for Improving Human Health
}

\author{
Diego F. Gomez-Casati, ${ }^{1}$ Marina Clemente, ${ }^{2}$ Alberto Inga, ${ }^{3}$ \\ Lucilia Saraiva, ${ }^{4}$ and Claudia P. Spampinato ${ }^{1}$ \\ ${ }^{1}$ Centro de Estudios Fotosintéticos y Bioquímicos (CEFOBI-CONICET), Universidad Nacional de Rosario, \\ S 2002 LRK Rosario, Argentina \\ ${ }^{2}$ UB6, Instituto de Investigaciones Biotecnológicas (IIB INTECH), Avenida Intendente Marino Km 8200, \\ CC 1647130 Chascomús, Argentina \\ ${ }^{3}$ Laboratory of Transcriptional Networks, Centre for Integrative Biology, University of Trento, 38123 Mattarello, Italy \\ ${ }^{4}$ REQUIMTE, Department of Biological Sciences, Laboratory of Microbiology, Faculty of Pharmacy, University of Porto, \\ 4050-313 Porto, Portugal
}

Correspondence should be addressed to Diego F. Gomez-Casati, gomezcasati@cefobi-conicet.gov.ar

Received 4 June 2012; Accepted 4 June 2012

Copyright (C) 2012 Diego F. Gomez-Casati et al. This is an open access article distributed under the Creative Commons Attribution License, which permits unrestricted use, distribution, and reproduction in any medium, provided the original work is properly cited.

In the last years there was an increase in the use of several organisms such as plants and yeast for medical and biotechnological purposes. Both organisms are very useful when research in humans is nonpossible or unfeasible due to ethical issues. There are several advantages of using model organisms such as the availability of genomic information, the possibility to easily make genetic manipulation and screenings, and availability of mutants in desired genes. In addition, these organisms are generally easy to grow.

The conservation in many cases of various metabolic and signaling pathways and basic cellular processes, often accompanied by proteomic and metabolomic information, is also an advantage in medical research, while the presence of specific metabolic pathways may be an advantage in the production of compounds of industrial interest. The paper of P. Jeandet et al. presents a detailed review on the biosynthesis, metabolism, and production of resveratrol, a stilbenic derivative. This compound is produced in the phenylalanine/polymalonate pathway, with the stilbene synthase (STS) being the key enzyme in this process. The increased use of resveratrol as "nutraceutical" (for nutrition and pharmaceutical uses) or in plant disease control makes its production an important goal in biotechnology. Different approaches to increase the production of this compound include the manipulation of the biosynthetic pathway and the overexpression of STS and other specific genes.
Besides the production of compounds of industrial interest, the use of plants for bioremediation and for the production of vaccine antigens is other important achievement in plant biotechnology related to improving human health. Some toxic compounds that can accumulate in plants are heavy metals. This is one of the biggest problems of environmental pollution and they also have severe consequences for their toxic effects on human health. The paper of Z. Zhang et al. presents a study of the accumulation of $\mathrm{Pb}$ and $\mathrm{Cd}$ in elite maize inbred lines. This study shows that there are significant differences in $\mathrm{Pb}$ and $\mathrm{Cd}$ accumulation between maize hybrid lines. So, it is possible to select low-metal accumulation lines for nutritional purposes. In addition, the study also showed the existence of maize lines with high capacity for accumulation of heavy metals that might be useful for bioremediation purposes.

On the other hand, plants become an emerging alternative for the production of vaccine antigens. The use of plants has several advantages such as easy gene engineering and low cost in the production of antigens. M. Clemente et al. reviewed the strategies for the production of antigens against malaria in plants. Malaria is a major health problem worldwide due to its high mortality rate, estimated at around one million people per year. Such strategies include the production of codon-optimized surface antigens of Plasmodium, the agent responsible for the disease, and the production 
of dual cholera-malaria antigens in tobacco plants and bacterial-made antigens.

There is also an extensive work using yeast, such as Saccharomyces cerevisiae and plants, mainly Arabidopsis thaliana, for the study of human pathologies. One example of this is the use of Saccharomyces for the study of the function of proteins of the human immunodeficiency virus (HIV) and the identification of its cellular partners common to yeast and humans. It is known that this retrovirus is the cause of AIDS, which affects more than 30 million people around the world. The paper of M. L. Andréola and S. Litvak provides a detailed review of the work done on the identification of cellular factors associated with different viral proteins, as well as the function and regulation of known enzymes encoded by the viral genome. The knowledge of the role of the viral proteins and cellular targets might allow the development of new therapeutic targets and strategies. In addition, this research could allow the discovery of new genes and proteins involved in virus proliferation and virus infection mechanisms, shortening the time necessary to develop different therapies against new viruses.

Besides the use of yeast, the study of plants, such as Arabidopsis thaliana, is a valuable tool in biomedicine, in the study of several mechanisms that might act similarly in producing human diseases, since human genes and proteins have been structurally and functionally conserved in plant organisms. The paper of C. P. Spampinato and D. F. Gomez-Casati presents a review of the current knowledge of human diseases of nuclear origin such as those related to defects in DNA repairing pathways and also mitochondrial pathologies, such as Friedreich's ataxia and respiratory complexes deficiencies, leading mainly to the production of neurological diseases. In addition, several studies in yeast could also contribute to uncover the etiology and pathogenesis of several neurodegenerative diseases as reviewed in the work of Pereira et al., including Alzheimer's, Parkinson's and Huntington's disease.

These papers represent an interesting compilation on the use of yeast and plants for biotechnological and biomedical purposes and the future prospects.

\section{Acknowledgments}

We thank all authors, reviewers, and the people of the Editorial of the Journal of Biomedicine and Biotechnology for the support to make possible the publication of this special issue.

Diego F. Gomez-Casati Marina Clemente Alberto Inga

Lucilia Saraiva

Claudia P. Spampinato 

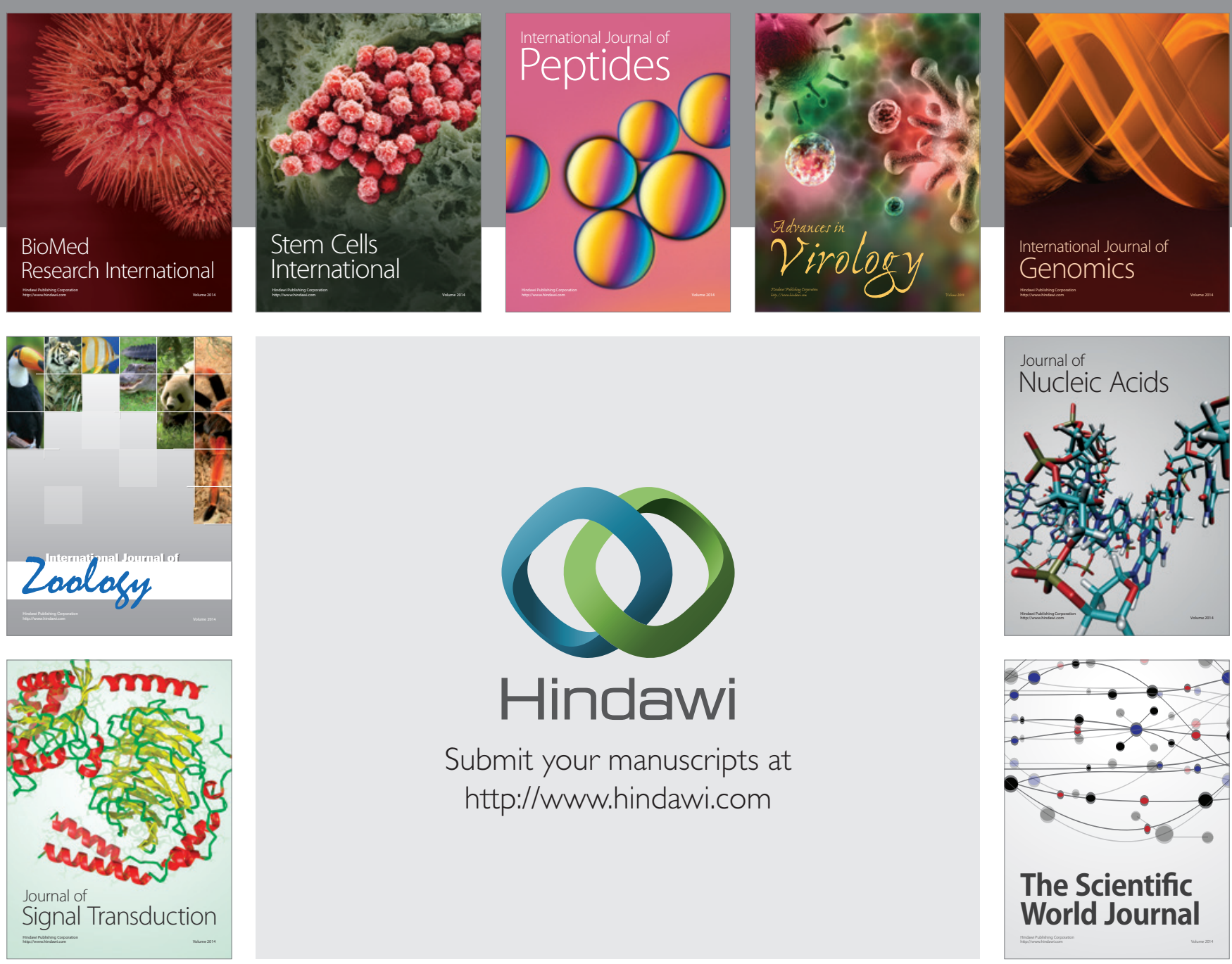

Submit your manuscripts at

http://www.hindawi.com
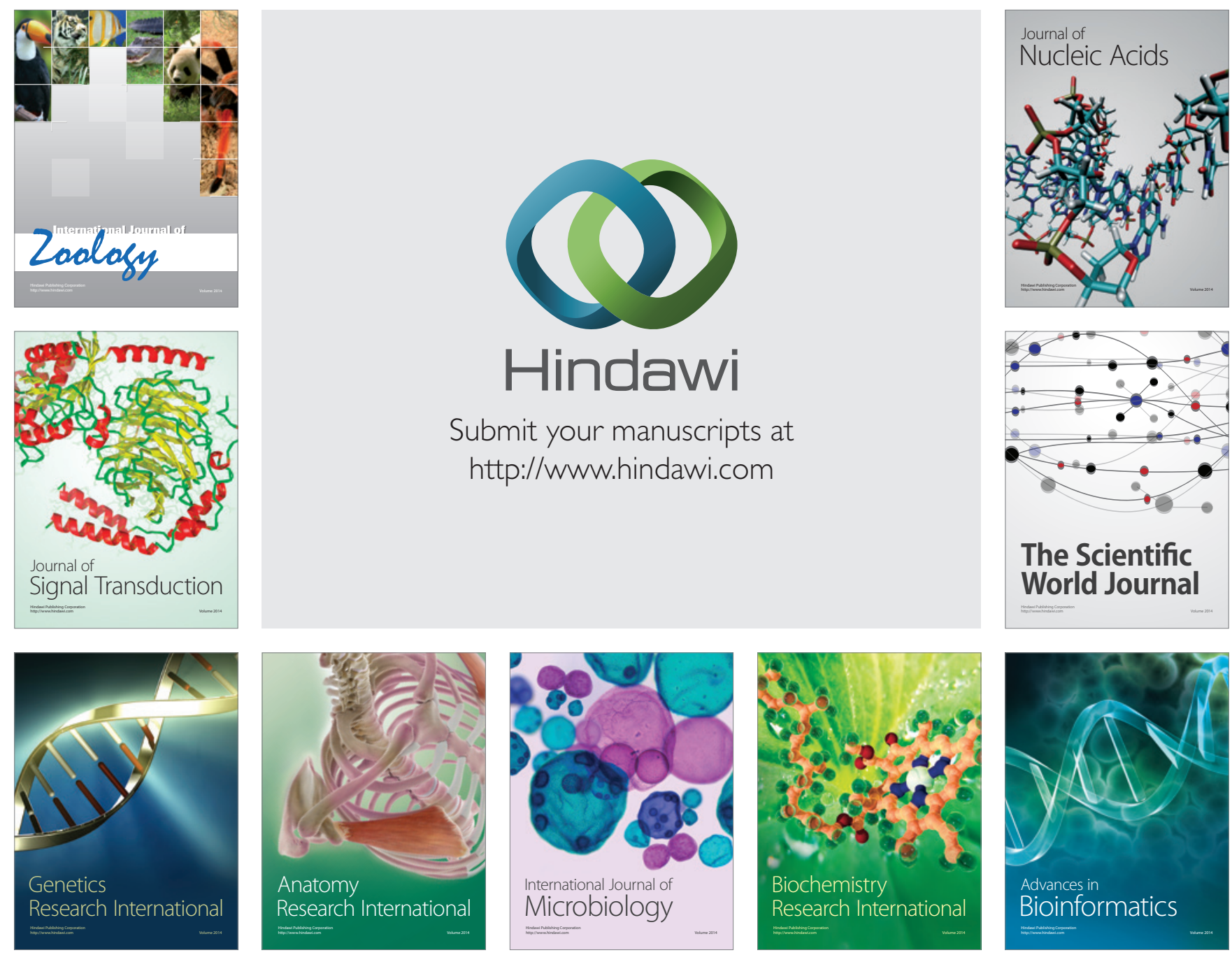

The Scientific World Journal
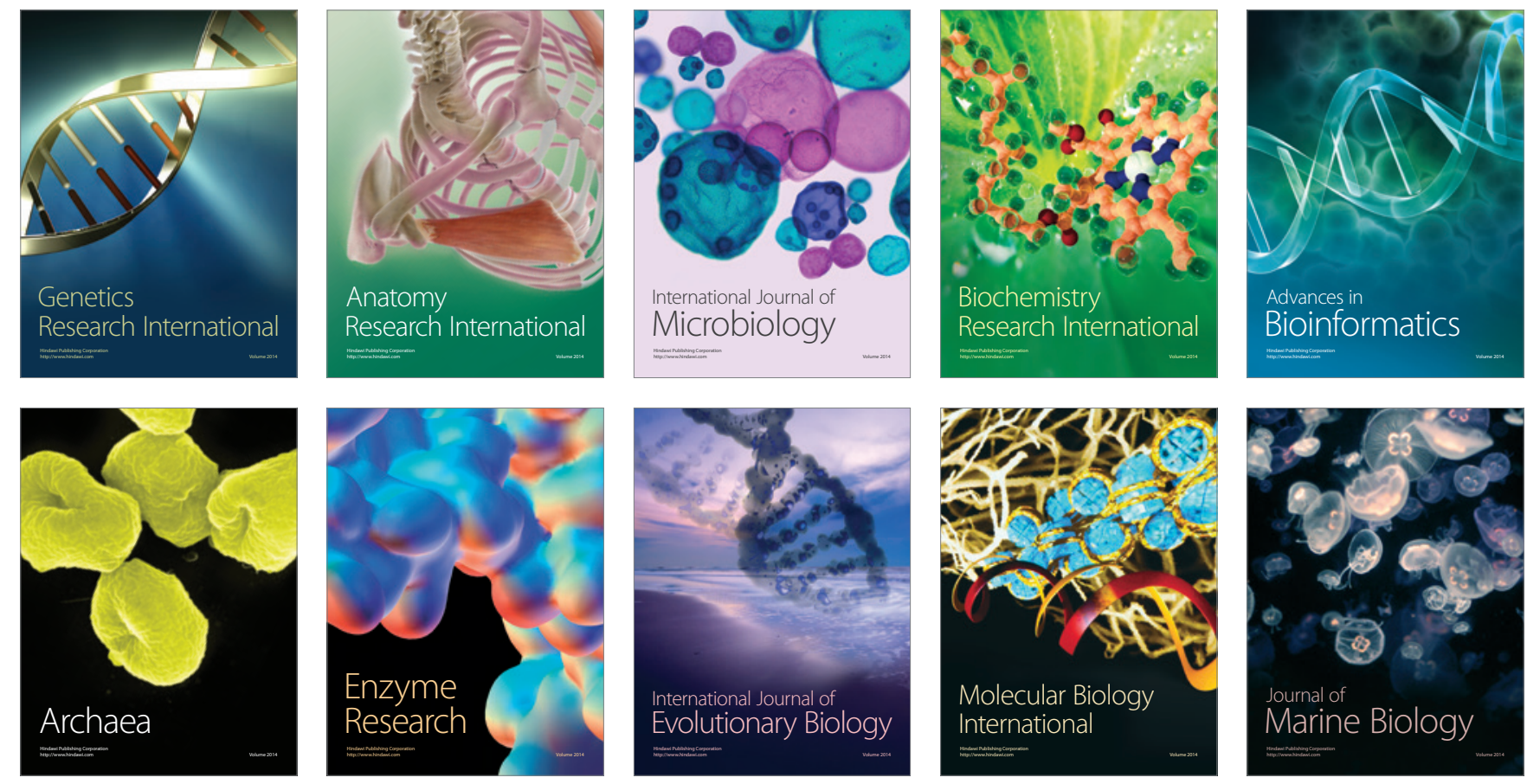